\title{
Macroinvertebrados del suelo y sus aportes a los servicios ecosistémicos, una visión de su importancia y comportamiento
}

\section{Soil macroinvertebrates and their contributions to ecosystem services, a vision about its importance and behavior}

\author{
TAPIA-CORAL, SANDRA ${ }^{1 *}$ Ph.D, TEIXEIRA, L. ANDREA² M.Sc, VELÁSQUEZ, ELENA ${ }^{3}$ Ph.D, \\ WALDEZ, FABIANO ${ }^{4} \mathrm{Ph} . \mathrm{D}$.
}

${ }^{1}$ Grupo de Pesquisa em Biologia, Ecologia e Função de Oligoquetos Terrestres (minhocas e enquitreídeos) Brasileiros. EMBRAPA Florestas, Brasil.

${ }^{2}$ Instituto Nacional de Pesquisas da Amazônia, Programa de Grande Escala Biosfera - Atmosfera INPA/LBA. Manaus, Brasil.

${ }^{3}$ Universidad Nacional de Colombia, Sede Palmira, Colombia. ${ }^{4}$ Instituto Federal de Educação, Ciência e Tecnologia do Amazonas (IFAM). Laboratório de Biologia do IFAM Campus Tabatinga, Brasil.

\section{Keywords:}

Agroforestry systems; land use;

management systems; soil conservation; soil fauna; rural smallholders.

\begin{abstract}
The conversion of natural forests into pastures or crops involves a drastic change, damaging in the natural forest succession and leading the systems for the early stages of immaturity, simplicity and instability. The degradation of ecosystems is associated with the loss of biodiversity and the ecosystem services it provides, therefore, it is necessary to know soil biodiversity, in order to generate knowledge and develop more sustainable practices. The goal was to determine the structure of communities of soil organisms and the soil nutrients and identify their contributions to ecosystem services in land use systems Tarumá-Mirim settlement, in the municipality of Manaus, Brazil. The greater abundance of macroinvertebrates soil was found in diversified agroforestry systems, which led to better conditions in the nutrients and the organic matter for to the colonization of soil organisms, mainly termite and earthworms, providing favorable chemical properties for the soil fertility and the increment of the nutrient cycling in the ecosystem.
\end{abstract}

\section{Palabras Clave:}

Sistema agroforestal; conservación del suelo; fauna de suelo; uso del suelo; sistemas de manejo; productores rurales.
INFORMACIÓN

Recibido: $18-10-2016$

Aceptado: 10-11-2016

Correspondência autor:

*sctcoral@gmail.com

\section{Resumen}

La conversión de los bosques naturales en pastizales o cultivos agrícolas implica un cambio drástico, dañando la sucesión forestal natural y llevando los sistemas a los primeros estadios de inmadurez, simplicidad e inestabilidad. La degradación de los ecosistemas está asociada a la pérdida de biodiversidad y a los servicios que esta provee, por lo tanto, es necesario conocer la biodiversidad del suelo, con el fin de generar conocimiento y desarrollar prácticas más sostenibles. El objetivo, fue determinar la estructura de las comunidades de organismos del suelo y nutrientes del suelo e identificar sus aportes a los servicios ecosistémicos en sistemas de uso del suelo del asentamiento Tarumá-Mirim, municipio de Manaus, Brasil. La mayor abundancia de macroinvertebrados del suelo fue encontrada en los sistemas agroforestales diversificados, que propiciaron mejores condiciones en nutrientes y materia orgánica para la colonización de los organismos del suelo, principalmente isópodos y lombrices, aportando mejores propiedades químicas para la fertilidad del suelo y un incremento del ciclaje de los nutrientes en el ecosistema. 


\section{Introducción}

Los suelos son un recurso esencial en múltiples servicios ecosistémicos que proveen beneficios a la población humana. Estos servicios ecosistémicos incluyen el suministro de nutrientes para las plantas, el mantenimiento de la estructura del suelo y la regulación del clima, y además un componente de la biodiversidad del suelo en los ecosistemas (ej. comunidad de macroinvertebrados) (MEA, 2005).

Los macroinvertebrados representan millones de especies importantes para el funcionamiento del suelo, incluyendo los llamados ingenieros del suelo - termitas, hormigas y lombrices - además de algunas especies de mil pies y escarabajos que modifican la estructura del suelo y la distribución de los recursos (especialmente materia orgánica) en el suelo para otros organismos, inclusive sobre las comunidades de microorganismos responsables de la mineralización y humificación, consumen cualquier clase de residuos orgánicos en asociación con la microflora del suelo (LAVELLE et al., 1997; LAVELLE et al., 2001).

Sin embargo, el recurso suelo se ve afectado por una excesiva explotación agrícola, ocasionando problemas de erosión por fuertes precipitaciones en suelos deforestados, además de pérdidas de componentes químicos, físicos y biológicos del mismo. Si éstos no son manejados de una manera adecuada, pueden representar un grave problema ambiental y productivo (ZWART, 2005).

El objetivo de este estudio, fue determinar la abundancia de los macroinvertebrados del suelo y la concentración de nutrientes en diversos sistemas de uso del suelo de productores rurales y su aporte como servicio ecosistémico.

\section{Materiales y métodos}

El trabajo fue realizado en el asentamiento TarumãMirim, localizado en el km 21 de la carretera BR174, Municipio de Manaus, Amazonas, Brasil (Figura 1).

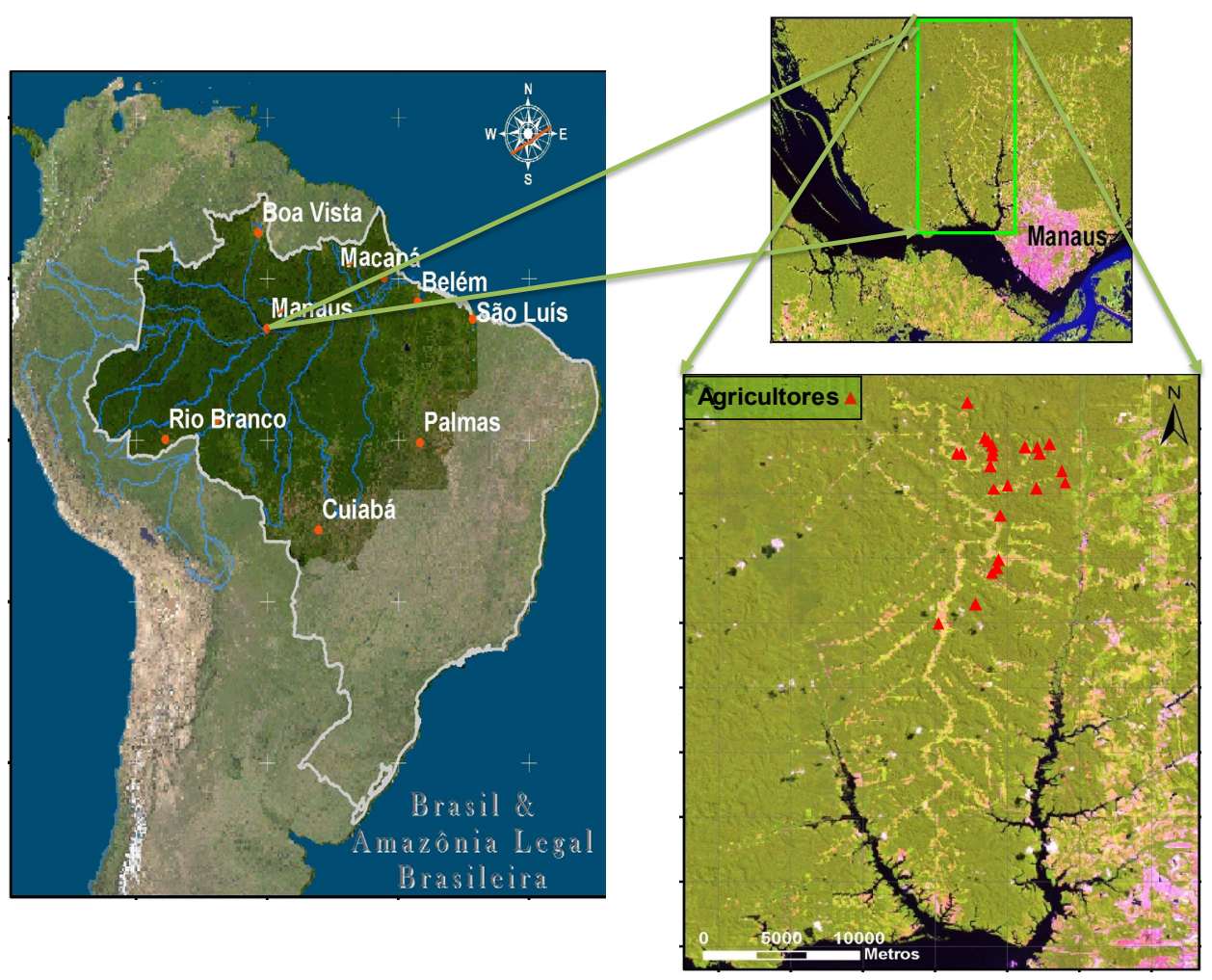

Figura 1. Localización del área de estudio, en el asentamiento Tarumã-Mirim, municipio de Manaus, Amazonas, Brasil. 
El asentamiento Tarumá-Mirim, tiene una extensión de 42.911 ha y fue creado por el Instituto Nacional de Colonización y Reforma Agraria - INCRA (Resolución $n^{\circ}$ 184/92 del 20/03/1992), con capacidad para albergar hasta 1.000 familias en lotes de 25 ha (INCRA, 1999). Para el presente estudio realizamos un "estudio de caso" con la participación de 10 familias de la "Asociación de Productores Rurales del Ramal de Pau Rosa" ASSAGRIR, los cuales tenían diversos sistemas de uso del suelo en sus lotes (SUS) (Tabla 1).

Tabla 1. Sistemas de uso del suelo (SUS) en las propiedades rurales de los agricultores familiares del asentamiento Tarumã-Mirim, Amazonas, Brasil.

\begin{tabular}{cc}
\hline $\begin{array}{c}\text { Sistemas de uso } \\
\text { del suelo (SUS) }\end{array}$ & Composición/Especies \\
\hline Monocultivo 1 & $\begin{array}{c}\text { Theobroma grandiflorum } \\
\text { Consorcio 1 }\end{array}$ \\
Consorcio 2 & $\begin{array}{c}\text { Inga edulis + Theobroma } \\
\text { grandiflorum }\end{array}$ \\
Sistema & $\begin{array}{c}\text { T. grandiflorum + Rollinia sp. + } \\
\text { Poraqueiba sericeaa + Annona } \\
\text { muricata + Carica papaya + Musa } \\
\text { Agroforestal-SAF }\end{array}$ \\
Bosque & Bosque primario manejado \\
\hline
\end{tabular}

El muestreo de macroinvertebrados del suelo, fue desarrollado con el método TSBF - Tropical Soil Biology and Fertility (ANDERSON e INGRAN, 1993), modificado para los SUS del asentamiento, es decir con bloques (monolitos) de suelo de 50 × $50 \mathrm{~cm}$ de largo y $20 \mathrm{~cm}$ de profundidad, debido a la presencia de lombrices grandes en el área. En cada sistema de uso del suelo, se muestrearon cinco monolitos de suelo a cada 10 metros a lo largo de un transecto de $50 \mathrm{~m}$ (Tabla 1). Cada monolito fue dividido en tres horizontes: hojarasca, $0-10 \mathrm{~cm}$ y $10-20 \mathrm{~cm}$ de profundidad. Los macroinvertebrados (animales visibles a simple vista $y \geq 2 \mathrm{~cm}$ de diámetro) fueron separados en el campo y conservados en frascos con alcohol al 96\%. Los muestreos, tuvieron la participación práctica de los productores rurales de cada propiedad, explicando previamente el trabajo de campo realizado y explicando los beneficios que los macroinvertebrados traen al suelo y su importancia para el crecimiento de las plantas que ellos tienen en sus sistemas (Figura 2).

Posteriormente, en el laboratorio, los macroinvertebrados del suelo fueron identificados al nivel de grandes grupos (orden-clase), siendo que las lombrices fueron identificadas a nivel de género y especie (cuando fue posible) por el especialista en lombrices Dr. Samuel James, de la Universidad de IOWA, USA.

El suelo de la primera capa $(0-10 \mathrm{~cm})$ del muestreo de TSBF, también sirvió para la caracterización química ( $\mathrm{pH}$ en agua, nitrógeno y carbono total, fosforo, potasio, calcio y magnesio), así como análisis de granulometría en cada sistema de uso del suelo, siguiendo la metodología de EMBRAPA (1997). Los resultados de los análisis químicos y granulométricos del suelo, son presentados en la Tabla 2.

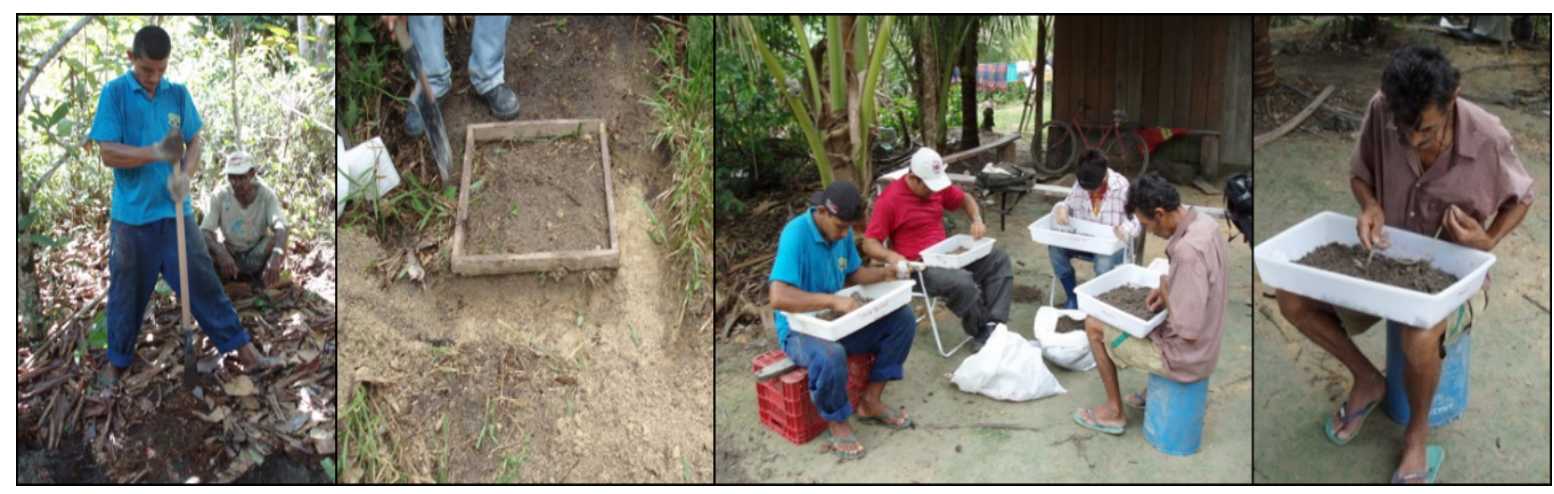

Figura 2. Muestreo de los macroinvertebrados del suelo en el asentamiento Tarumã-Mirim, Amazonas, Brasil, con participación de los productores rurales de cada propiedad. 
Tabla 2. Características químicas y granulometría de los diferentes sistemas de uso del suelo en la capa de 0-10 $\mathrm{cm}$ del asentamiento Tarumã-Mirim.

\begin{tabular}{|c|c|c|c|c|c|c|c|c|c|c|c|}
\hline \multirow[b]{2}{*}{ Sistemas } & \multirow{2}{*}{$\begin{array}{c}\mathrm{pH} \\
\left(\mathrm{H}_{2} \mathrm{O}\right)\end{array}$} & $\mathbf{N}$ & C & MO & $\mathbf{P}$ & K & $\mathrm{Ca}$ & Mg & Arcilla & Arena & Limo \\
\hline & & \multicolumn{3}{|c|}{ (\%) } & \multicolumn{4}{|c|}{ g.kg-1 } & \multicolumn{3}{|c|}{$(\%)$} \\
\hline Monocultivo & 4.27 & 1.55 & 27.55 & 47.5 & 2.79 & 0.10 & 0.01 & 0.04 & 85.38 & 9.02 & 5.60 \\
\hline Consorcio1 & 4.11 & 1.45 & 29.21 & 50.37 & 2.39 & 0.14 & 0.02 & 0.05 & 80.36 & 15.04 & 4.60 \\
\hline Consorcio2 & 4.11 & 1.45 & 29.21 & 50.37 & 2.39 & 0.14 & 0.02 & 0.05 & 80.36 & 15.04 & 4.60 \\
\hline SAF & 4.34 & 2.02 & 35.75 & 61.64 & 2.97 & 0.14 & 0.03 & 0.10 & 85.14 & 11.08 & 3.78 \\
\hline Bosque & 3.92 & 1.95 & 33.76 & 58.2 & 2.59 & 0.06 & 0.03 & 0.09 & 65.65 & 21.48 & 12.87 \\
\hline
\end{tabular}

Análisis de los datos: Los datos fueron analizados (variables de macroinvertebrados) mediante análisis de componentes principales (ACP) y un test de Montecarlo, con el propósito de identificar los factores predominantes para la densidad y comparar las comunidades de los macroinvertebrados en los diferentes SUS (VELÁSQUEZ et al., 2007). Para los análisis estadísticos se utilizó el software R (R CORE TEAM, 2013) y el paquete ade4 para el análisis multivariado (DRAY y DUFOUR, 2007).

\section{Resultados}

Macroinvertebrados del suelo: En total fueron colectados 12.964 individuos de 20 ordenes. Una diversidad de 17 grupos fue encontrada en el sistema consorcio 2, seguido del SAF (15), consorcio 1 (14), monocultivo (13) y bosque (10).

El SAF presentó la densidad más alta por $\mathrm{m}^{2}$ con $6.924 \mathrm{ind} / \mathrm{m}^{2}$, siendo, $3.096 \mathrm{ind} / \mathrm{m}^{2}$ individuos de Isopoda, $1.528 \mathrm{ind} / \mathrm{m}^{2}$ de Oligochaeta y $724 \mathrm{ind} / \mathrm{m}^{2} \mathrm{de}$ Isoptera. El área con menor densidad fue el bosque, con un total de $784 \mathrm{ind} / \mathrm{m}^{2}$, formado principalmente por Isoptera (368 ind $\left./ \mathrm{m}^{2}\right)$ y Oligochaeta $\left(192 \mathrm{ind} / \mathrm{m}^{2}\right)$ (Figura 3).

El análisis de componentes principales (ACP) presentó diferencia significativa $(p: 0,06)$ en la separación de los sistemas de uso del suelo en función de los valores de los indicadores (Figura 4). El primer factor explica el $17,3 \%$ de la variabilidad de los datos y separa principalmente el bosque con los valores más bajos de todos los indicadores. El segundo factor explica el (13\%) y separa al SAF con los valores más altos de los indicadores de macroinvertebrados del suelo. Este sistema presentó además los valores más altos de abundancia y diversidad de los macroinvertebrados (Figura 4).

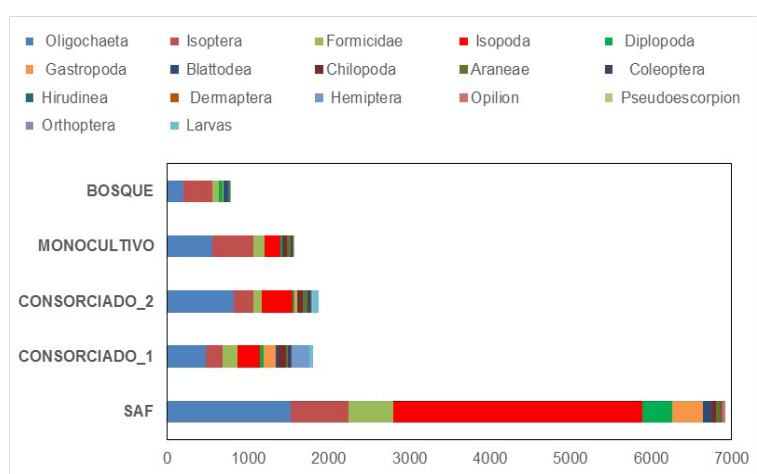

Figura 3. Promedio de los macroinvertebrados del suelo (ind $\mathrm{m}^{-2}$ ) en los diferentes sistemas de uso del suelo (SUS) de los productores rurales del asentamiento Tarumã-Mirim, Manaus, Amazonas, Brasil.

$\mathrm{Al}$ analizar las variables químicas de los nutrientes del suelo y granulometría por medio del análisis de componentes principales fue explicada con $63,75 \%$ en los primeros factores y significativa en los diferentes sistemas de uso del suelo a través de la prueba de Monte Carlo $(p<0.5)$ (Figura 5). No en tanto, el análisis de coinercia de las variables de macroinvertebrados del suelo y los nutrientes, no detectó correlaciones significativas.

Diversidad de Oligochaeta (lombrices de tierra): Fueron identificados seis géneros de lombrices y la especie exótica Pontoscolex corethrurus en todos los SUS muestreados. El sistema consorcio 2 y el SAF presentaron la mayor abundancia de los oligoquetos, todos de la familia Glossoscolecidae. Siendo que Glossodrilus sp.1 y sp. 2, fueron los más predominantes en el consorcio 2 y SAF respectivamente (Tabla 2). P. corethrurus, fue encontrado en todos los sistemas de uso del suelo, inclusive en el bosque, aunque en menores proporciones y fueron muy predominantes en los sistemas de monocultivo y SAF (Tabla 3 ). 


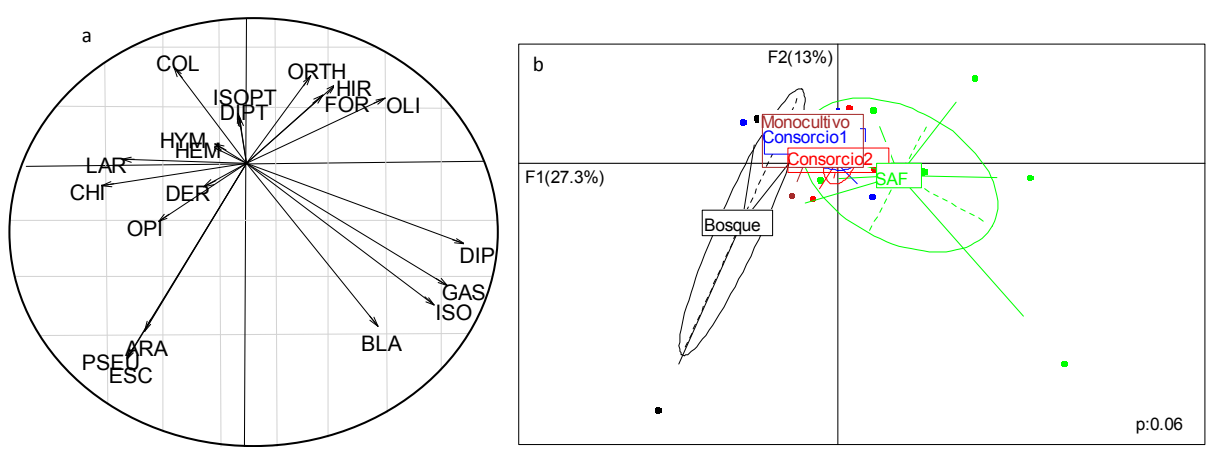

Figura 4. PCA de los indicadores de macroinvertebrados del suelo. (a) circulo de correlación de las variables: ARA (Araneae), BLA(Blattodea), CHI (Chilopoda), COL (Coleoptera), DER (Dermaptera), DIP (Diplopoda), DIP (Diptera), ESC (Escorpionida), FOR (Formicidae), GAS (Gasteropoda), HEM (Hemiptera), HIR (Hirudinea), HYM (Hymenoptera), ISO (Isopoda), ISOPT (Isoptera), LAR (larvas de macroinvertebrados), OLI (Oligochaeta), OPI (Opilionida), ORTH (Orthoptera), PSEU (Pseudoescorpionida). (b) Proyección de los sistemas de uso del suelo en función de los indicadores.

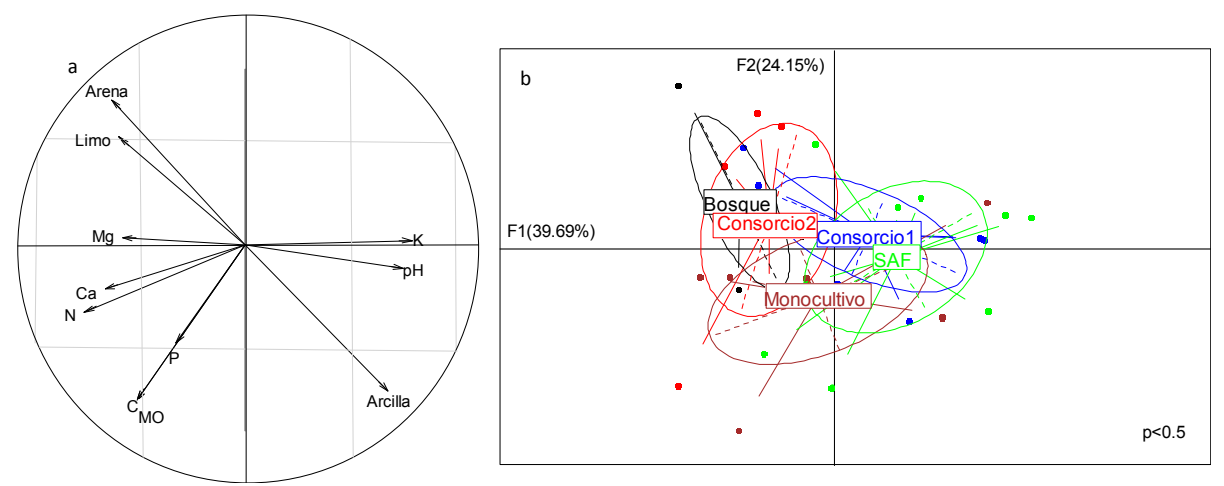

Figura 5. PCA de los indicadores de nutrientes y granulometría del suelo. (a) círculo de correlación de las variables (b) Proyección de los sistemas de uso del suelo en función de los indicadores.

Tabla 3. Diversidad de oligoquetos (lombrices) en los sistemas de uso del suelo del asentamiento Tarumã-Mirim, Amazonas, Brasil.

\begin{tabular}{cccccc}
\hline Género/Especie & Monocultivo & Consorcio 1 & Consorcio 2 & SAF & Bosque \\
\hline Glossoscolex 1 & $5( \pm 2)$ & $17( \pm 11)$ & 0 & $9( \pm 6)$ & 0 \\
Glossoscolex 2 & $53( \pm 22)$ & $21( \pm 10)$ & 0 & $15 \pm(12)$ & $5( \pm 2)$ \\
Glossodrilus 1 & $29( \pm 12)$ & $3( \pm 1)$ & $31( \pm 11)$ & $73( \pm 42)$ & $4( \pm 2)$ \\
Glossodrilus 2 & $11( \pm 2)$ & $9( \pm 7)$ & $112( \pm 70)$ & $14( \pm 8)$ & $25( \pm 16)$ \\
Rhinodrilus sp. & 0 & $3( \pm 2)$ & $1( \pm 1)$ & 0 & 0 \\
Urobenus sp. & $12( \pm 7)$ & $12( \pm 11)$ & 0 & $6( \pm 5)$ & $4( \pm 3)$ \\
Pontoscolex corethrurus & $54( \pm 10)$ & $8( \pm 5)$ & $29( \pm 10)$ & $44( \pm 19)$ & $5( \pm 2)$ \\
\hline
\end{tabular}


La variación en la diversidad de lombrices en los sistemas de uso del suelo fue explicada con $44,4 \%$ en los dos factores de los análisis de PCA. Diferencias significativas en la diversidad de lombrices en los sistemas de uso del suelo fue comprobada a través de la prueba de Monte Carlo $(p<0.5)$ (Figura 6). La variancia de los nutrientes del suelo (C, N, P, K, Ca y Mg) fue explicada con el $69.6 \%$ en los dos factores del PCA, siendo también significativa los nutrientes en los diferentes sistemas de uso del suelo a través de la prueba de Monte Carlo $(p<0.5)$. Sin embargo, el análisis de coinercia, de la diversidad de lombrices y los nutrientes del suelo, no se detectó correlaciones significativas en los sistemas de uso del suelo.

\section{Discusión}

Los macroinvertebrados del suelo, tienen un papel importante en la provisión de diferentes servicios ecosistémicos, mediante su acción sobre los procesos del suelo (BARROS et al., 2008). Estos procesos dependen de la composición de los macroinvertebrados del suelo y el conocimiento de las actividades humanas sobre estas comunidades de organismos del suelo. En el asentamiento Taruma-Mirim, la quema de bosque primario y secundario es frecuente para la producción de carbón, siendo una de las principales actividades económicas realizada por los moradores principalmente (TAPIA-CORAL et al., 2008).

Todos los sistemas de uso del suelo evaluados del presente trabajo, tuvieron mayores densidades de los macroinvertebrados de suelo, que los diversos agroecosistemas reportados por BARROS et al. (2008), LIMA et al. (2010) y TAPIA CORAL et al. (2014). Los sistemas agroforestales del asentamiento Tarumã-Mirim presentaron una composición de especies de plantas muy diversa (Tabla 1), siendo importante para la presencia de una mayor abundancia de los macroinvertebrados del suelo en este sistema, indicando de esta manera que la variedad florística influyó en la abundancia y diversidad de los macroinvertebrados del suelo. Así mismo, los SAF presentaron mayores concentraciones de cantidades de carbono, materia orgánica y contenido de nutrientes.

Los sistemas agroforestales, son sistemas conservacionistas en razón de su semejanza con sistemas naturales (BARROS et al., 2008), inclusive, recientemente los sistemas agroforestales diversificados fueron recomendados con el objetivo de recuperar áreas degradadas, en el departamento del Caquetá, Colombia, debido a su "conexión" entre los componentes naturales del bosque y las áreas de producción agroforestal que inciden de manera positiva en el mantenimiento del suelo y el control de plagas en los cultivos (LAVELLE, 2012). El grupo Isopoda fue el más abundante en los SAF del asentamiento TarumãMirim, probablemente porque el suelo de este sistema estuvo siempre cubierto de abundante materia orgánica, lo que ocasiona mejores condiciones climáticas y alimento disponible en razón de la mayor composición de especies de plantas en este sistema. Resultados similares, fueron encontrados anteriormente por TAPIACORAL et al. (2005) en sistemas agroforestales diversificados con abundante materia orgánica esparcida en el sistema y que terminan siendo

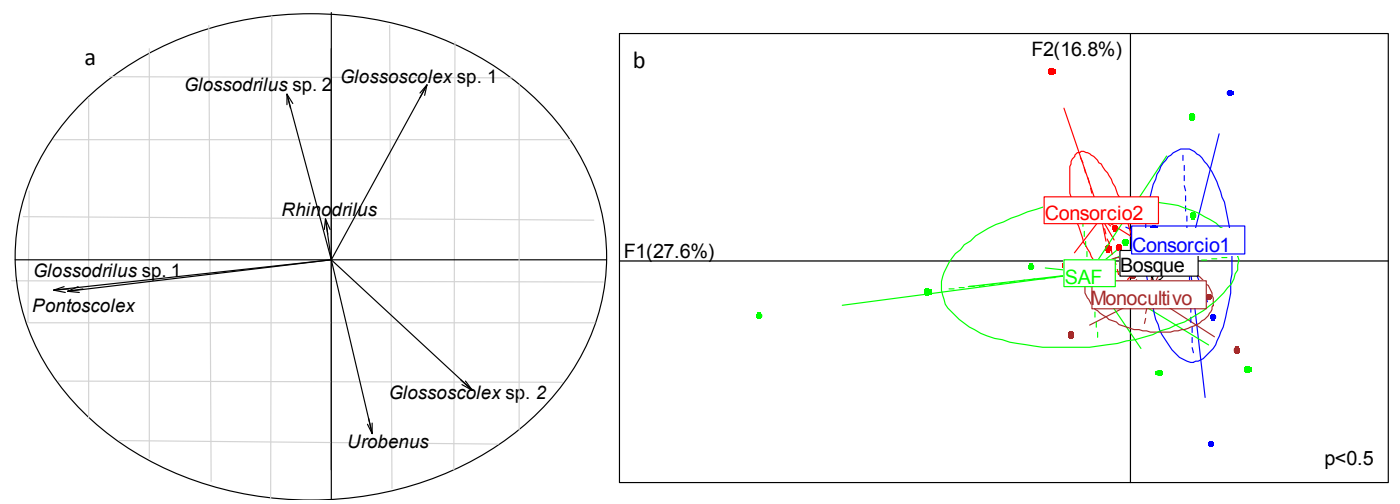

Figura 6. PCA de los indicadores. (a) circulo de correlación de las variables de oligoquetos. (b) Proyección de los sistemas de uso del suelo en función de los indicadores de oligoquetos. 
hábitats importantes para la colonización de los isópodos fragmentadores de hojarasca.

La menor contribución de los bosques en densidad y diversidad, posiblemente, estuvo influenciada por el uso y manejo constante del bosque por parte de los agricultores del asentamiento TarumãMirim que realizan en cada propiedad, como la recolección permanente de frutos de pan llevar, leña para uso doméstico y sobre todo por el corte de árboles para la producción de carbón vegetal con el fin de obtener ingreso inmediato (TAPIACORAL et al., 2008). Sin embargo, la diversidad del bosque contribuyó para el hallazgo de grupos importantes indicadores de la diversidad como los grupos de Escorpionida, Pseudoescorpionida y Areneae, que generalmente son encontrados en bosques naturales y poco perturbados. En todos los sistemas de uso del suelo fueron encontrados a los llamados "ingenieros del suelo" (lombrices, termitas y hormigas) principalmente en los SAF y sistemas de consorcio 1 y 2 , siendo que estos grupos en particular afectan directa o indirectamente el recurso disponible para otras especies, a través de la modificación física de materiales bióticos o abióticos y pueden ser considerados como grupos clave de bioindicadores del suelo (JONES et al., 1994).

Agradecimientos: Agradecemos a la "Fundación de Amparo a Pesquisa do Estado do Amazonas" - FAPEAM por el financiamiento del proyecto (Proceso 2207/05). Un agradecimiento especial a los agricultores de la "Asociación de Agricultores del Ramal de Pau Rosa" - ASSAGRIR, cuya participación activa hizo posible la realización de este estudio.

\section{Referencias}

ANDERSON, J.M.; INGRAM, J.S. 1993. Tropical Soil Biology and Fertility: A Handbook of Methods. 2 ed., CAB International, Wallingford, UK.

BARROS, E.; MATHIEU, J.; TAPIA-CORAL, S.; NASCIMENTO, A.R.L.; LAVELLE, P. 2008. Comunidades da Macrofauna do Solo na Amazônia Brasilera. Págs. 171-191. En: Moreira, F.M.S.; Siqueira, J.O.; Brussaard, L. (Eds.) Soil Biodiversity in Amazonian and other Brazilian Ecosystems. CABI Publishing.

DRAY, S.; DUFOUR, A.B. 2007. The ade4 package: implementing the duality diagram for ecologists. J Stat Softw $22(4): 1-20$.

EMBRAPA. 1997. Centro Nacional de Pesquisa de Solos. Manual de métodos de análise de solo. $2^{a}$ ed. Rio de Janeiro, RJ. (EMBRAPA - CNPS. Documentos; 1). Brasil.

INCRA. 1999. Informações do Projeto de Assentamento Tarumã-Mirim. Manaus, Brasil.

JONES, C.G.; LAWTON, J.H.; SHACHAK, M. 1994. Organisms as ecosystems engineers. Oikos (69): 373-386.

LAVELLE, P.; BIGNELL, D.; LEPAGE, M.; WOLTERS, V.; ROGER, P.; INESON, P.; HEAL, O.W.; DHILLION, S. 1997. Soil function in a changing world: the role of invertebrate ecosystem engineers. European Journal of Soil Biology (33):159-193.

LAVELLE, P; BARROS, E; BLANCHART, E; BROWN, G; DESJARDINS, T; MARIANI, L. ; ROSSI, J. 2001. SOM management in the tropics: Why feeding the soil Macrofauna? Nutrient Cycling in Agroecosystems $61(1-2): 53-61$.

LAVELLE, P. 2012. Servicios ambientales del Caquetá desaparecerán en diez años. Disponible en: http:// www.unperiodico.unal.edu.co/vpp/article/servicioambiental. Consultado: 08-09-2012. 
LIMA, S.S. de.; AQUINO, A.M. de.; LEITE, L.F.C.; VELASQUÉZ, E.; LAVELLE, P. 2010. Relação entre a macrofauna edáfica e atributos químicos do solo em diferentes agroecossistemas. Pesquisa Agropecuaria Brasileira 45 (3):322-331.

MILLENIUM ECOSYSTEM ASSESSMENT, 2005. Ecosystems and Human Well-being: Synthesis, Island Pre. ed. Washington, DC.

R CORE TEAM (2013) R: a language and environment for statistical computing. R Foundation for Statistical Computing. Vienna, Austria. http://www.R-project.org/

TAPIA-CORAL, S.C.; LUIZÃO, F.; WANDELLI, E.; FERNANDES, E.C.M. 2005. Carbon and nutrient stocks in the litter layer of agroforestry system in central Amazonia, Brazil. Agroforestry Systems (65):33-42.

TAPIA-CORAL, S.C.; COSTA, J.R.; CHOTA, I.J.; MORAIS, J.W.; WANDELLI, E.; LUIZÃO, F. 2008. Serviços Ambientais em ecossistemas manejados por agricultores familiares do Assentamento TarumãMirim, Amazonas. 1. ed. Manaus: INPA. Manaus, Brasil.

TAPIA-CORAL, S.C.; LUIZÃO, F.; PASHANASI, B.; DEL CASTILLO, D.; LAVELLE, P. 2014. Influência da massa e nutrientes da liteira sobre a composição dos macro-invertebrados em plantíos florestais na Amazônia peruana. Folia Amazónica 23 (2):171-186.

VELÁSQUEZ, E.; LAVELLE, P.; ANDRADE, M. 2007. GISQ, a multifunctional indicator of soil quality. Soil Biology and Biochemistry (39):3066-3080.

ZWART, M; ROJO, J; DE LA CRUZ, R.Y. 2005. Coberturas y la salud del suelo. Tierra Tropical 1 (1):9-20. 